\title{
Estado ecológico del humedal urbano Phuyuqocha - Cajonahuaylla, San Jerónimo - Cusco - Perú
}

\section{Ecological state of urban wetland Phuyuqocha - Cajonahuaylla, San Jerónimo - Cusco - Perú}

\author{
Percy Yanque-Yucra ${ }^{1 *}$, María O.Arce-Ttito ${ }^{1} \&$ Yan C. Y. Nina-Loayza ${ }^{1}$

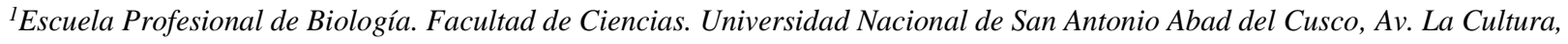 \\ 733 Cusco, Perú. \\ *Autor corresponsal: Percy Yanque-Yucra, percy.yanque@unsaac.edu.pe
}

\begin{abstract}
RESUMEN
El presente trabajo se desarrolló en el humedal urbano Phuyuqocha - Cajonahuaylla, Cusco. El objetivo fue evaluar el estado ecológico del humedal. Se consideró cuatro indicadores: batimétricos y morfométricos, biológicos (fitoplancton, macroinvertebrados acuáticos, macrófitas, fauna superior), microbiológicos (Coliformes termo tolerantes) e indicadores físicos y químicos del agua. La metodología corresponde a la propuesta de Rico, 2006. La evaluación batimétrica y morfométrica muestra una profundidad máxima de $1,07 \mathrm{~m}$, lecho irregular, superficie de espejo de agua $4108,78 \mathrm{~m}^{2}$, volumen de $4109.88 \mathrm{~m}^{3}$. Considerando el protocolo seguido, la valoración de los indicadores morfométricos califica como muy malo, los biológicos muy malo, los indicadores físicos y químicos malo y los indicadores microbiológicos moderado; la valorización general de todos los indicadores alcanza un promedio de 1.07 puntos, el mismo que corresponde a un estado ecológico general malo. Las causas de degradación del humedal, que reducen su estado ecológico, corresponden a la presencia de residuos sólidos, presencia de animales domésticos, arrojo de desmontes, cerco de protección deteriorado entre otros.
\end{abstract}

Palabras Clave: Humedal urbano, estado ecológico, contaminación, indicadores biológicos.

\section{ABSTRACT}

The present work was developed in the urban wetland Phuyuqocha - Cajonahuaylla, Cusco. The objective was to evaluate the ecological status of the wetland. Four indicators were considered: bathymetric and morphometric, biological (phytoplankton, aquatic macroinvertebrates, macrophytes, higher fauna), microbiological (thermo-tolerant coliforms) and physical and chemical indicators of water. The methodology corresponds to the proposal of Rico, 2006. The bathymetric and morphometric evaluation shows a maximum depth of $1.07 \mathrm{~m}$, irregular bed, surface of a water mirror $4108.78 \mathrm{~m}^{2}$, volume of $4109.88 \mathrm{~m}^{3}$. Considering the protocol followed, the evaluation of the morphometric indicators qualifies as very bad, the biological ones very bad, the physical and chemical indicators bad and the microbiological indicators moderate; the general valuation of all the indicators reaches an average of 1.07 points, the same that corresponds to a poor general ecological state. The causes of wetland degradation, which reduce its ecological status, correspond to the presence of solid waste, the presence of domestic animals, dumping of clearings, deteriorated protection fence, among others.

Key Words: Urban wetland, ecological status, pollution, biological indicators.

\section{INTRODUCCIÓN}

La Convención de Ramsar define a los Humedales como: "extensiones de marismas, pantanos y turberas, o superficies cubiertas de aguas, sean éstas de régimen natural o artificial, permanentes o temporales, estancadas o corrientes, dulces, salobres o saladas, incluidas las extensiones de agua marina cuya profundidad en marea baja no exceda de seis metros" (Secretaría de la Convención de Ramsar, 2018). La clasificación de la Convención reconoce a los humedales, por el tipo de ambiente donde se presentan y la geoforma, tres ambientes generales: I) humedales marinos/costeros, II) Humedales interiores (continentales) y III) humedales construidos por el hombre. En los humedales se establecen centros de desarrollo económico cuyo eje es la industria y los transportes, aunados a la producción de alimentos y el turismo (Secretaría de la Convención de Ramsar, 2018).

El estado ecológico es una expresión de la calidad de la estructura y el funcionamiento de los ecosistemas acuáticos asociados a las aguas superficiales (Ruza, 2008).

El humedal Phuyuqocha - Cajonahuaylla, es el único ecosistema urbano de la ciudad del Cusco, Perú; este humedal presenta una fuerte presión antrópica, vinculada a la inadecuada gestión de los residuos sólidos y aguas servidas, la falta de educación ambiental, construcción de un acceso vehicular a la sede de la policía ecológica, construcción de una vía asfaltada
Cusco-Urcos, construcción de viviendas en sus alrededores, que están generando problemas sobre el humedal acelerando su procesos de envejecimiento y eutrofización hasta un punto crítico de extinguirse por completo (Paredes, 1998).

Esta información permitirá que las entidades públicas y actores sociales tengan un punto de referencia en la toma de decisiones que permitan su recuperación, manejo sostenible, protección, preservación y conservación de este humedal. El objetivo fue, evaluar el estado ecológico del humedal urbano Phuyuqocha - Cajonahuaylla, San Jerónimo - Cusco.

\section{METODOLOGÍA}

La investigación considero el humedal Cajonahuaylla, seleccionado debido a su localización y cercanía a entidades urbanas que han experimentado un importante crecimiento en las últimas décadas; por lo que, esta rodeado de edificaciones diversas que descargan aguas residuales directamente e influyen en la calidad hidromorfologica y ecológica de la misma (Paredes, 1998).

Este humedal conocido también como Phuyuqocha, se localiza en las coordenadas UTM $188680 \mathrm{~m} \mathrm{E}, 188920 \mathrm{~m}$ E y $8500120 \mathrm{~m} \mathrm{~N}, 8500320 \mathrm{~m} \mathrm{~N}$ de la zona 19L, a una altitud de 3213 m, próximo a la prolongación Av. De La Cultura, sur-este de la provincia del Cusco, distrito San Jerónimo, Región Cusco, abarca una superficie de 1.36 ha (Fig. 1). 

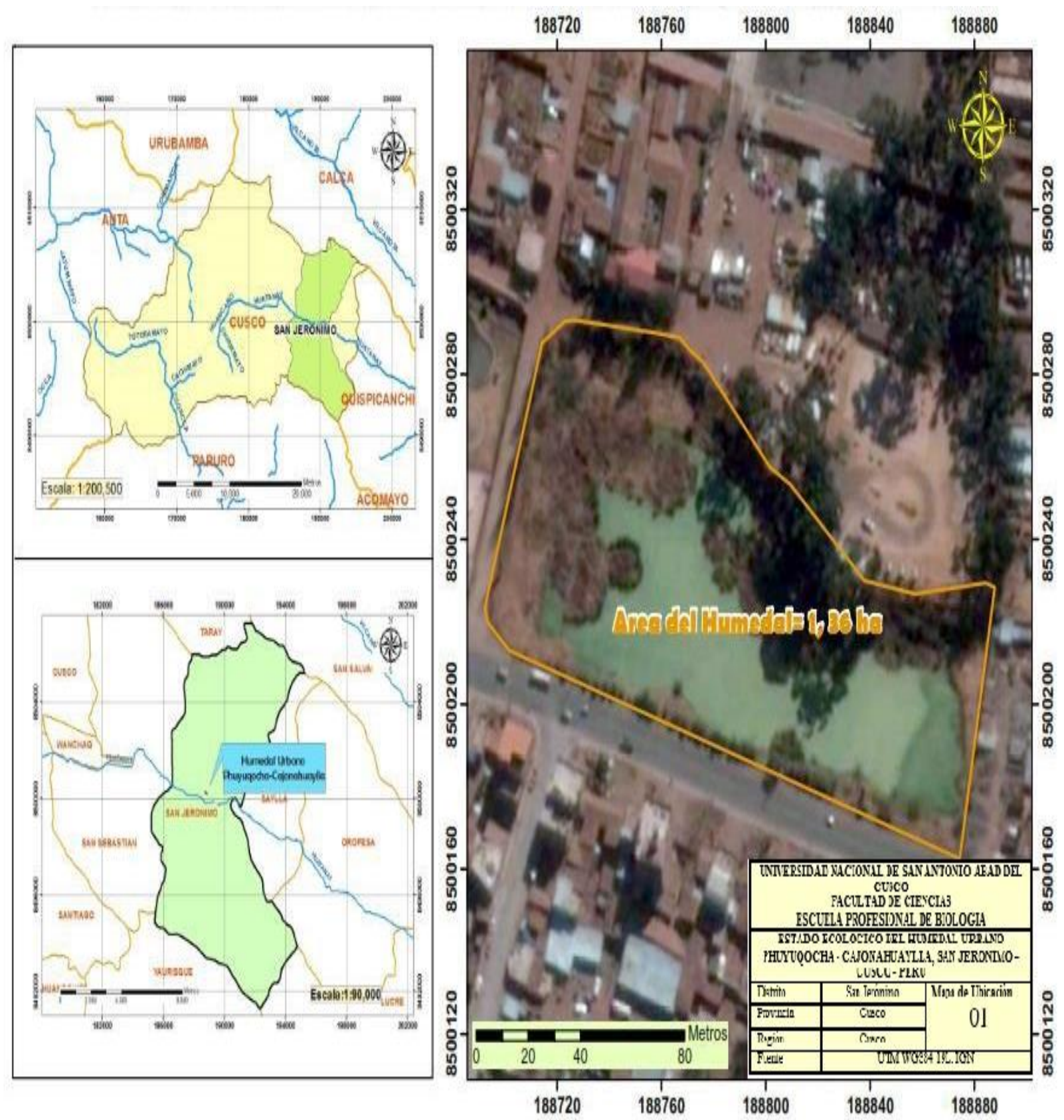

Figura 1. Ubicación del humedal urbano Cajonahuaylla - Phuyucocha en el distrito de San Jerónimo, Cusco.

La investigación es de tipo descriptiva - transversal. Por la cantidad de variables analizadas, los muestreos fueron de forma aleatoria para ciertos parámetros, sistemática en algunos casos y preferencial en otros; así mismo, considerando que es un humedal pequeño (1.36 ha) permitió evaluar, en algunos casos, todo el humedal. El muestreo se realizó durante cuatro meses entre la época de lluvia y la temporada de mínima inundación de 2017, para generar una evaluación rápida del estado actual de su condición ecológica en la temporada en donde se registra la menor variabilidad espacial debido a las escorrentías. La características morfométricas y batimétricas fueron levantadas recurriendo a los sistemas de información geográfica y el software ArcGis 10.4. El diseño de muestreo incluyó tres sitios de muestreo, donde se tomó muestras de agua, macrofitas, macrofauna bentónica y fitoplanton (las variables bióticas se determinaron en base al protocolo propuesto por Samanez et al. (2014). Para calificar la calidad del agua con respecto a la normatividad peruana vigente se cuantificaron varios parámetros físicos, químicos y biológicos (Tabla 1). Las muestras de agua fueron analizadas en laboratorio dentro de los tiempos establecidos por las técnicas y normas pertinentes. La estimación del Estado Ecológico se llevó a cabo a través de la ponderación de cuatro indicadores (Tabla 01), cada uno considera diferentes subindicadores definidos de acuerdo a la propuesta de Rico (2006). Realizadas las evaluaciones, se procedió al llenado de diferentes matrices, elaboradas para cada subindicador que permitió el reporte del estado de calidad y puntuación correspondientes (Rico, 2006). 
Tabla 1. Parámetros, subindicadores y rangos de calidad para humedales

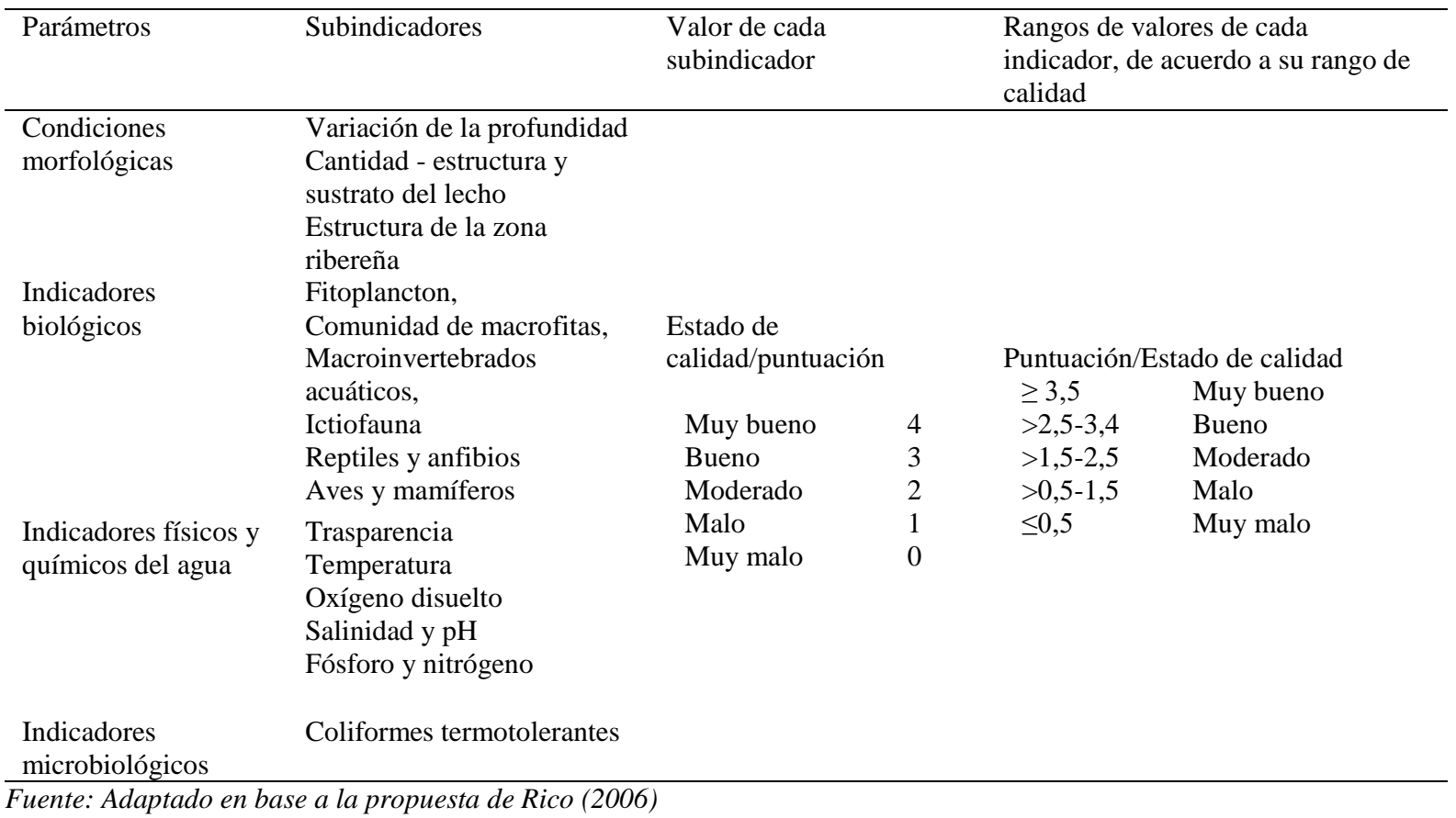

El primer paso fue calcular para estos indicadores de calidad de los estados de aquellos indicadores parciales. El procedimiento se basa en la media de las puntuaciones de los estados parciales:

Puntuación indicador $=$ Media de las puntuaciones parciales .

Finalmente, para la determinación del estado ecológico global se consideró básicamente el estado del grupo de indicadores biológicos, determinando de forma directa el estado ecológico si su estado es muy malo, malo o moderado. En el caso de que los indicadores biológicos tengan un estado bueno o muy bueno, se tendrá en cuenta si el estado de los indicadores físicos y químicos y/o morfológicos presentan un estado inferior al bueno; así, si los indicadores biológicos tuvieran un estado muy bueno el estado ecológico del humedal sería bueno, y en el caso de que tuvieran un estado bueno el humedal sería moderado.

Conocidos los estados de los cuatro grupos de indicadores de calidad, se procedió a determinar del estado ecológico global directamente cruzando el estado de calidad del grupo de indicadores biológicos, con el peor de los estados de calidad de entre los indicadores físico, químicos y morfológicos según la Tabla 2

Tabla 2. Escala de colores y caracterización del estado ecológico general

\begin{tabular}{|c|c|c|c|c|c|c|}
\hline \multirow{2}{*}{\multicolumn{2}{|c|}{ Estado Ecologico }} & \multicolumn{5}{|c|}{ Estado hidromorfologicos o estado indicadores fisico quimicos (peor de los estados) } \\
\hline & & Muybueno & Bueno & Moderado & Malo & Muymalo \\
\hline \multirow{3}{*}{$\begin{array}{l}\text { Estado } \\
\text { Indicadore }\end{array}$} & Murbueno & Mup bueno & Muy bueno & Bueno & Moderado & Moderado \\
\hline & Bueno & Bueno & Bueno & Moderado & Moderado & Moderado \\
\hline & Moderado & Moderado & Moderado & Moderado & Moderado & Moderado \\
\hline \multirow[t]{2}{*}{ Biologicos } & Malo & Malo & Malo & Malo & Malo & Malo \\
\hline & Muymalo & Muymalo & Muymalo & Muymalo & Murmalo & Muy malo \\
\hline
\end{tabular}

\section{RESULTADOS}

Condiciones Batimétricas y Morfométricas del Humedal

Las evaluaciones del humedal Phuyuqocha - Cajonahuylla permitió hacer una representación de la topografía subacuática; información con la cual se pudo determinar diferentes parámetros que lo caracterizan (Fig. 2).

La profundidad máxima fue $1,07 \mathrm{~m}$. resultando ser un cuerpo lentico somero, las isobatas están separas a $0,05 \mathrm{~m}$ evidenciando la forma del relieve topográfico del humedal. El lecho es irregular a lo largo de toda su extensión.

Existen tres parámetros que necesariamente tienen que ser reportados y categorizados:

La variación de profundidad es malo/muy malo considerando que el humedal es receptor directo de desechos orgánicos, inorgánicos y el efecto de la presión antrópica se manifiesta a más de $50 \%$, generando el incremento de sedimentos en su lecho.

La cantidad, estructura y sustrato del lecho, es muy malo, por las remociones o vertidos de sustratos (aguas residuales) que afectan a más del $1 \%$ de la superficie del humedal.

La estructura de la zona ribereña, presenta alteraciones físicas constantes por acumulación de residuos sólidos, llantas, animales en descomposición, presencia de especies exóticas, invasión de plantas emergentes como la totora; estas condiciones permiten calificarla como muy malo.

\section{Fitoplancton e Índice Trófico Planctónico}

Se determinó 15 géneros pertenecientes a cuatro divisiones. Los géneros reportados, son característicos de organismos asociados a ambientes eutrofizados, siendo más abundantes Staurosira y Oscillatoria.

En base al análisis cuantitativo de Fitoplancton y otras variables se ha determinado el Índice Trófico Planctónico ITP que alcanza un valor de 16 por lo que fue ubicado dentro de los intervalos de calificación como muy malo (Tabla 3 ). 
Tabla 3. Análisis cuantitativo de Fitoplancton

\begin{tabular}{llc}
\hline División & Género & $\mathrm{N}^{\circ}$ de org/l \\
\hline Cyanophyta & Oscillatoria & 24,2 \\
& Spirulina & 2,2 \\
Euglenophita & Phacus & 2,2 \\
& Epithemia sp1 & 6,6 \\
& Straurosira & 74,8 \\
& Sinedra & 13,2 \\
& Actinocyclus & 2,2 \\
& Diatoma & 2,2 \\
Bacillarophyta & Epithemia sp2 & 2,2 \\
& Denticula & 2,2 \\
& Ankira & 2,2 \\
& Cyclotella & 2,2 \\
& Ligmophora & 2,2 \\
& Navicula & 4,4 \\
Chlorophyta & Scenedesmus & 2,2 \\
& Cosmarium & 2,2 \\
\hline
\end{tabular}

\section{Comunidad Macrofítica}

Se determinaron seis especies correspondientes a cinco familias taxonómicas. La familia Cyperaceae es dominante con las especies Scirpus americanus Pers. y Schoenoplectus californicus ssp. Tatora (Kunth) T. koyama (Tabla 4).

Tabla 4. Vegetación flotante y emergente

\begin{tabular}{|c|c|c|}
\hline Familia & Especie & $\begin{array}{c}\text { Tipo de } \\
\text { vegetación }\end{array}$ \\
\hline Araceae & Lemna gibba L. & Flotante \\
\hline Azollaceae & Azolla filiculoides Lam. & Flotante \\
\hline Araliaceae & $\begin{array}{l}\text { Hydrocotyle ranunculoide } \\
\text { L. }\end{array}$ & smergente \\
\hline Brassicaceae & $\begin{array}{l}\text { Rorippa nasturtium- } \\
\text { aquaticum (L.) Hayek. }\end{array}$ & Emergente \\
\hline \multirow[t]{2}{*}{ Cyperaceae } & Scirpus americanus Pers. & Emergente \\
\hline & $\begin{array}{l}\text { Schoenoplectus } \\
\text { californicus ssp. Tatora } \\
\text { (Kunth) T. koyama }\end{array}$ & Emergente \\
\hline Juncaceae & Juncus arcticus Willd. & Emergente \\
\hline Scrophulariaceae & $\begin{array}{l}\text { Veronica anagallis- } \\
\text { aquatica } L .\end{array}$ & Emergente \\
\hline
\end{tabular}

Se determinó 59 especies de flora circundante pertenecientes a 31 familias. La familia más abundante en número de especies es Asteraceae (Tabla 5).

\section{Cobertura de la flora}

El humedal urbano presenta siete zonas con diferentes formaciones vegetales, la superficie del ecosistema lentico está cubierto mayormente por Lemna gibba, Azolla filiculoides, Hidrocotyle ranunculoides. De acuerdo a los intervalos de valoración es calificada como moderada (Tabla 6).

Tabla 5. Cantidad de especies de la vegetación circundante

\begin{tabular}{lclc}
\hline Familia & $\begin{array}{c}\text { Número de Familia } \\
\text { especies }\end{array}$ & $\begin{array}{c}\text { Número } \\
\text { de } \\
\text { especies }\end{array}$ \\
\hline Amaranthaceae & 2 & Oleaceae & 1 \\
Amaryllidaceae & 1 & Onagraceae & 1 \\
Anacardiaceae & 1 & Oxalidaceae & 1 \\
Apiaceae & 1 & Passifloraceae & 1 \\
Asteraceae & 7 & Pinaceae & 1 \\
Berberidaceae & 1 & Plantaginaceae & 1 \\
Brassicaceae & 6 & Poaceae & 5 \\
Bromeliaceae & 1 & Polemoniaceae & 1 \\
Cactaceae & 2 & Polygalaceae & 1 \\
Cucurbitaceae & 1 & Polygonaceae & 1 \\
Cupressaceae & 1 & Rosaceae & 3 \\
Cyperaceae & 1 & Salicaceae & 2 \\
|Ephedraceae & 1 & Scrophulariaceae & 1 \\
Fabaceae & 5 & Solanaceae & 5 \\
Malvaceae & 1 & Typhaceae & 1 \\
Myrtaceaae & 1 & Verbenaceae & 1 \\
\hline
\end{tabular}

Tabla 6. Cobertura vegetal del humedal

\begin{tabular}{lrc}
\hline Descripción & Área (m2) & $\begin{array}{c}\text { Porcentaj } \\
\mathrm{e}\end{array}$ \\
\hline $\begin{array}{l}\text { Espejo de agua cubierto por Lemna } \\
\text { gibba y Azolla filiculoides }\end{array}$ & 4112,22 & $30 \%$ \\
$\begin{array}{l}\text { Área invadida por totoral } \\
\text { Área invadida por Hydrocotyle } \\
\text { ranunculoides }\end{array}$ & 3585,00 & $26 \%$ \\
$\begin{array}{l}\text { Área cubierta por Pennisetum } \\
\text { clandestinum }\end{array}$ & 688,28 & $5 \%$ \\
$\begin{array}{l}\text { Área forestada con } \\
\begin{array}{l}\text { Polylepis racemosa, Salix } \\
\text { humboltiana y Populus nigra }\end{array}\end{array}$ & 2563,00 & $19 \%$ \\
$\begin{array}{l}\text { Área cubierta por matorral arbustivo de } \\
\text { valle seco interandino }\end{array}$ & 1753,57 & $13 \%$ \\
$\begin{array}{l}\text { Área cubierta por Eucaliptus globulus } \\
\text { Total }\end{array}$ & 261,10 & $2 \%$ \\
\hline
\end{tabular}

\section{Macroinvertebrados acuáticos}

Estos macroinvertebrados, están representados por 13 familias pertenecientes a seis clases. Las clases más abundantes son Insecta, Gasterópoda y Turbellaria. De acuerdo a los criterios de calificación, la riqueza de taxonómica es muy malo por haber reportado menos de 15 familias (Tabla 7).

\section{Riqueza Taxonómica de aves acuáticas y terrestres}

Se determinó 12 especies entre acuáticas, terrestres, residentes y visitantes, pertenecientes a 11 familias. Anser anser domesticus es una especie domestica de propiedad de la policía ecológica en el humedal. Según los criterios de calificación, la riqueza taxonómica de la ornitofauna es malo (Tabla 8). 
Tabla 7. Riqueza taxonómica de macroinvertebrados

\begin{tabular}{llll}
\hline Phylum & Clase & Orden & Familia \\
\hline $\begin{array}{l}\text { Platyhelminthes } \\
\text { Annelida }\end{array}$ & Turbellaria & Tricladida & Planariidae \\
Mollusca & Hirudinea & Arhynchobdelida & Clossiphoelidae \\
& Bivalvia & Veneroida & Sphaeridae \\
& Gasteropoda & Basommatophora & $\begin{array}{l}\text { Physidae } \\
\text { Planorbidae }\end{array}$ \\
Arthropoda & Insecta & Coleoptera & Carabidae \\
& & Elmidae \\
& & Culicidae \\
& & Diptera & Muscidae \\
& & Dixidae \\
& & Notenectidae \\
& & Hyalellidae \\
\hline
\end{tabular}

Tabla 8. Diversidad de aves acuáticas y terrestres

\begin{tabular}{|c|c|c|}
\hline Familia & Especie & Condición \\
\hline Anatidae & Anser anser domesticus & acuática \\
\hline Ardeidae & Nycticorax nycticorax & acuática \\
\hline Rallidae & Gallinula chloropus & acuática \\
\hline Scolopacidae & Actitis macularius & acuática \\
\hline \multicolumn{2}{|c|}{ ThreskiornithidaePlegadis ridwayi } & acuática \\
\hline Carduelidae & Carduellis magellanica & terrestre \\
\hline Columbidae & Patagioenas maculosa & terrestre \\
\hline Columbidae & Zenaida auriculata & terrestre \\
\hline Emberizidae & Zonotrichia capensis & terrestre \\
\hline Thraupidae & Phrygilus punensis & terrestre \\
\hline Trochilidae & Patagona gigas & terrestre \\
\hline Turdidae & Turdus chiguanco & terrestre \\
\hline
\end{tabular}

\section{Riqueza taxonómica de ictiofauna, anfibios, reptiles y mamíferos}

Según la categoría para la calificación de la riqueza taxonómica de ictiofauna se encuentra en la categoría de malo/muy malo ausencia total de poblaciones de ícticas.

No se encontró presencia de anfibios y reptiles en el área, de acuerdo a los criterios de calificación la riqueza taxonómica de anfibios y reptiles es muy mala. La riqueza taxonómica de mamíferos calificó como muy malo, debido a la ausencia de mamíferos silvestres.

\section{Indicadores Físicos y Químicos}

Transparencia. El valor alcanzado fue $0,14 \mathrm{~m}$ por la existencia de gran cantidad de partículas suspendidas lo que imposibilita el ingreso de luz, calificó en la categoría muy malo. Temperatura. Dentro del humedal alcanzo $17^{\circ} \mathrm{C}$, sin embargo, la temperatura atmosférica fue $29^{\circ} \mathrm{C}$, (variación anual de $2.5^{\circ} \mathrm{C}$ ) las categorías de calificación de este parámetro correspondieron a malo/muy malo.
Oxígeno disuelto. La concentración fue $0.8 \mathrm{mg} / \mathrm{L}$, por debajo de los estándares de calidad ambiental para agua (D.S. $\mathrm{N}^{\circ}$ 015-2015-MINAM), categoría 4 (Conservación de un ambiente acuático). La calificación del oxígeno disuelto fue malo/muy malo.

Conductividad eléctrica. Este parámetro fue $590 \mu \mathrm{S} / \mathrm{cm}$ aceptable dentro de los estándares de calidad ambiental de agua (D.S. $\mathrm{N}^{\circ}$ 015-2015- MINAM), que se encuentra categorizado como moderado.

Potencial de hidrogeniones. fue 6.8, valor que se encuentra dentro de los Estándares de Calidad Ambiental para Agua, Categoría 4. Enmarcados dentro de los valores de calificación como moderado.

Fosforo total. El análisis dio un resultado de $0,3 \mathrm{mg} / \mathrm{l}$, indicando un nivel no excesivo, este parámetro calificó como muy malo. Nitrógeno total. El contenido no es muy elevado, el valor fue $0.4 \mathrm{mg} / \mathrm{l}$ y presentó una calificación de muy bueno.

\section{Indicadores microbiológicos}

Los análisis de coliformes termotolerantes dieron un valor de $1500 \mathrm{NMP} / 100 \mathrm{ml}$, valor que sobrepasan los Estándares de Calidad Ambiental para Agua (D.S. N ${ }^{\circ} 015-$ 2015-MINAM), categoría 4 (Conservación de un ambiente acuático); por consiguiente, existe vertidos humanos al humedal; dentro de la categorización ésta se ubica dentro de moderado.

\section{Estado Ecológico General del Humedal Urbano}

Los indicadores batimétricos y morfométricos tienen un estado ecológico de muy malo, al igual que los indicadores biológicos a excepción de la cobertura vegetal con un estado moderado debido a las condiciones químicas del agua que favorecen el surgimiento de la vegetación.

Los indicadores físicos y químicos tienden a presentar estados muy malos, a excepción de la acidificación y salinización. Los indicadores microbiológicos tienen una calificación de moderado evidenciando que el humedal está siendo afectado por actividades antrópicas por vertido de aguas residuales.

La valoración de los cuatro indicadores permitió establecer que el estado ecológico general del humedal urbano Phuyuqocha -_Cajonahuaylla es malo (Tabla 9). 
Tabla 9. Valoración del estado ecológico del humedal urbano Puyococha - Cajonahuaylla

\begin{tabular}{|c|c|c|c|c|c|c|c|}
\hline $\begin{array}{l}\text { Grupo de } \\
\text { Indicadores }\end{array}$ & Indicadores & Indicador parcial & Val. & $\begin{array}{c}\text { Estado de } \\
\text { calidad } \\
\text { ecológica }\end{array}$ & $\begin{array}{l}\text { Promedio } \\
\text { de } \\
\text { valoración }\end{array}$ & $\begin{array}{c}\text { Estado } \\
\text { Ecológico } \\
\text { del Indicador }\end{array}$ & $\begin{array}{l}\text { Estado } \\
\text { Ecológico } \\
\text { General }\end{array}$ \\
\hline \multirow{3}{*}{$\begin{array}{l}\text { Indicadores } \\
\text { Batimetricos } \\
\text { y } \\
\text { Morfometricos }\end{array}$} & \multirow{3}{*}{$\begin{array}{l}\text { Condiciones } \\
\text { morfológicas }\end{array}$} & Variación de la profundidad & 0.5 & Muy malo & \multirow{3}{*}{0.5} & \multirow{3}{*}{ Muy malo } & \multirow{18}{*}{ Malo } \\
\hline & & $\begin{array}{l}\text { Cantidad, estructura y } \\
\text { sustrato del lecho }\end{array}$ & 0 & Muy malo & & & \\
\hline & & $\begin{array}{c}\text { Estructura de la zona } \\
\text { ribereña }\end{array}$ & 0 & Muy malo & & & \\
\hline \multirow{7}{*}{$\begin{array}{l}\text { Indicadores } \\
\text { Biológicos }\end{array}$} & Fitoplancton & ITP & 0.5 & Muy malo & \multirow{6}{*}{0.5} & \multirow{7}{*}{ Muy malo } & \\
\hline & $\begin{array}{l}\text { Comunidad } \\
\text { macrofitica }\end{array}$ & Cobertura vegetal & 2 & Moderado & & & \\
\hline & $\begin{array}{l}\text { Macroinvertebrados } \\
\text { acuaticos }\end{array}$ & Riqueza taxonómica & 0 & Muy malo & & & \\
\hline & \multirow{4}{*}{ Fauna } & $\begin{array}{l}\text { Riqueza Taxonómica de } \\
\text { ictiofauna }\end{array}$ & 0.5 & Muy malo & & & \\
\hline & & $\begin{array}{l}\text { Riqueza Taxonómica de } \\
\text { anfibios y reptiles }\end{array}$ & 0.5 & Muy malo & & & \\
\hline & & $\begin{array}{l}\text { Riqueza Taxonómica de } \\
\text { avifauna }\end{array}$ & 0 & Muy malo & & & \\
\hline & & $\begin{array}{l}\text { Riqueza Taxonómica de } \\
\text { mamíferos }\end{array}$ & 0 & Muy malo & \multirow{8}{*}{1.28} & & \\
\hline \multirow{7}{*}{$\begin{array}{l}\text { Indicadores } \\
\text { Físicos y } \\
\text { Químicos }\end{array}$} & Transparencia & & 0 & Muy malo & & \multirow{7}{*}{ Malo } & \\
\hline & Temperatura & & 0.5 & Muy malo & & & \\
\hline & Oxígeno disuelto & & 0.5 & Muy malo & & & \\
\hline & Acidificación $(\mathrm{pH})$ & & 2 & Moderado & & & \\
\hline & Salinización (Condu & tibilidad eléctrica) & 2 & Moderado & & & \\
\hline & \multirow{2}{*}{ Nutrientes } & Fosfato total & 0 & Muy malo & & & \\
\hline & & Nitrógeno total & 4 & Muy bueno & & & \\
\hline $\begin{array}{l}\text { Indicadores } \\
\text { Microbiol. }\end{array}$ & \multicolumn{2}{|c|}{ Coliformes termotolerantes (NMP/100 ml) } & 2 & Moderado & 2 & Moderado & \\
\hline
\end{tabular}

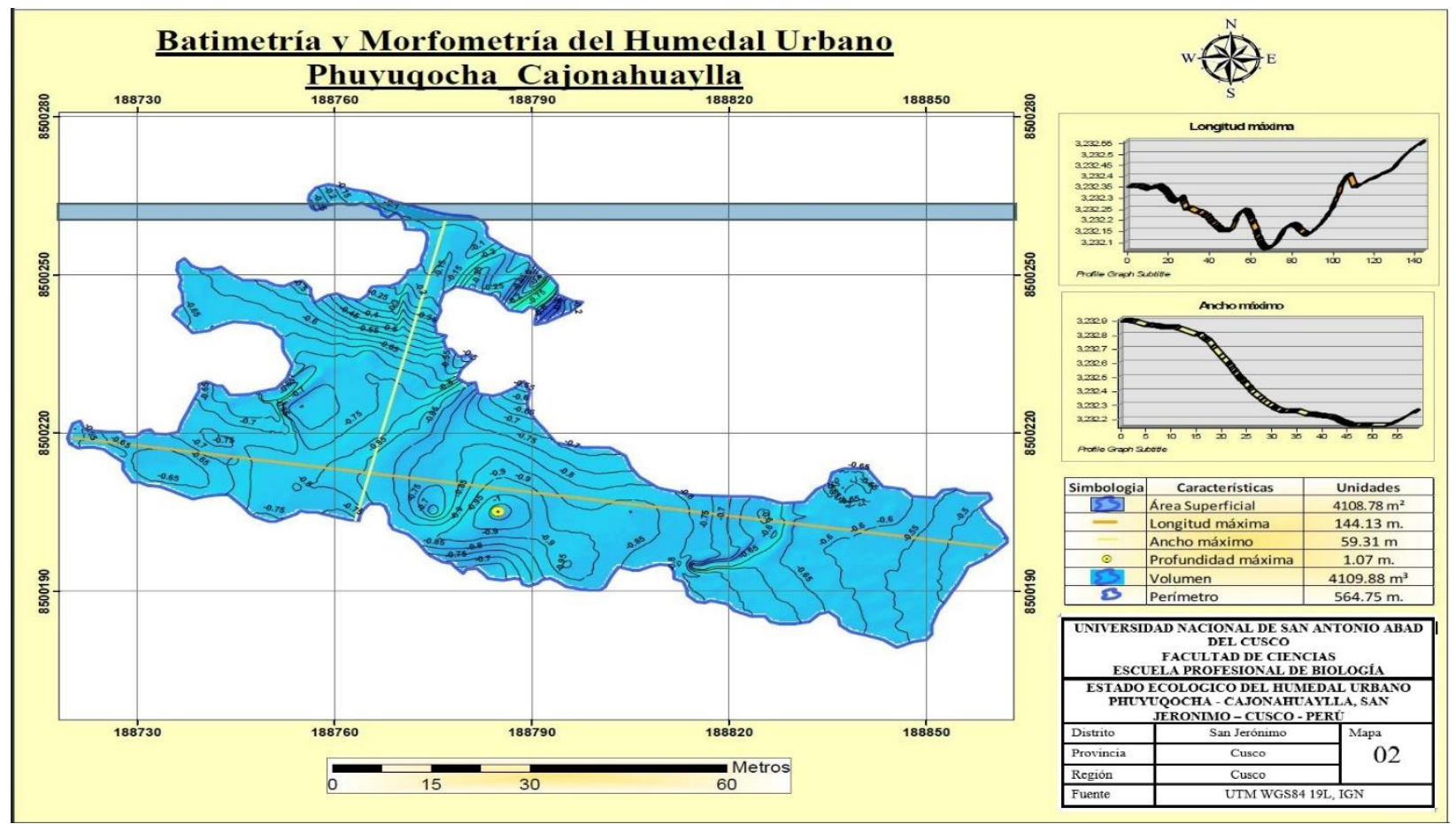

Figura 2. Batimetría y morfometría de humedal urbano Cajonahuaylla - Phuyucocha 


\section{DISCUSIONES}

El máximo de profundidad del humedal urbano fue 1,07 $\mathrm{m}$. Rico (2006) menciona que si la profundidad es menor a 3 $\mathrm{m}$, la luz puede llegar hasta el fondo y permitir una mayor extensión e incluso ocupación total de las comunidades litorales.

En este último caso el ciclo de nutrientes sufre un cambio sustancial, ya que, se establece una competencia entre los productores primarios del bentos y plancton, viéndose favorecido el primero, de forma que estos sistemas aunque son eutróficos, presentan aguas claras durante el periodo de crecimiento de macrófitos sumergidos, la profundidad a la que penetre la luz del Sol aporta la energía para la fotosíntesis y determinará la profundidad a la cual las plantas acuáticas pueden crecer. Por otro lado las condiciones morfométricas, especialmente la variación de la profundidad de acuerdo a las categorías de calificación del estado ecológico, fue malo/muy malo debido a que el humedal es receptor indirecto de desechos orgánicos, inorgánicos y el efecto de la presión antrópica se manifiesta a más de 50\%, generando el incremento de sedimentos en su lecho, remociones o vertidos de sustratos que afectan a más del $1 \%$ de la superficie del humedal o existen canales de drenaje que consiguen la desecación total del humedal y según la estructura ribereña existen alteraciones físicas permanentes del litoral que superan el $25 \%$ de la línea rivereña (Rico, 2006).

El Índice Trófico Planctónico fue de 16, está ubicado dentro de los intervalos de calificación como muy malo, esto indica que la colonización vegetal del humedal se suele organizar según la diferente profundidad, siendo parte de esta el fitoplancton que estando en menor cantidad hay menor presencia de clorofila " $a$ " en el agua o su equivalencia la biomasa Fitoplanctónica, está fuertemente ligada al proceso de la productividad primaria (Tucto, 2014) y es responsable directa de la salud del ecosistema (Rico, 2006).

La riqueza taxonómica de macroinvertebrados acuáticos es muy malo por la cantidad de familias $<15$, representa poca diversidad, con requerimientos ecológicos diferentes relacionados con las características hidromorfológicas, físicas, químicas y biológicas del medio acuático; además, indica que hay alteraciones a mediano y largo plazo, ya que sus especies poseen ciclos de vida entre menos de un mes hasta más de un año. En el ámbito de aplicación de la Directiva Marco del Agua de la Unión Europea, los invertebrados bentónicos se consideran útiles para la detección y seguimiento de las presiones físicas y químicas relacionadas con la contaminación térmica, cambios en la mineralización del agua, contaminación orgánica, eutrofización, contaminación por metales u otros contaminantes (Rico, 2006).

Indicadores físicos del agua como la transparencia, fue menor a $1 \mathrm{~m}$, indica mucha cantidad, composición y tamaño de los materiales en suspensión, estas partículas suspendidas en el agua reducen la profundidad a la cual la luz puede penetrar. Como segundo indicador temperatura, con una variación anual de $2.5^{\circ} \mathrm{C}$, representa el poco desarrollo de los diversos procesos que en ella se realizan, ya que no se modifica la solubilidad de las sustancias, disminuyendo la de los sólidos disueltos y gases (Roider, 1998). Existen variaciones normales de temperatura dependiendo de la hora del día y la noche, el grado de turbidez del agua es uno de ellos, ya que las partículas en suspensión captan más eficazmente el calor y lo transfieren al cuerpo de agua (Flores, 2007).

Indicadores químicos del agua, como oxígeno disuelto muestra una concentración de $0.8 \mathrm{mg} / \mathrm{l}$, que está por debajo de los estándares de calidad ambiental (D.S. $\mathrm{N}^{\circ}$ 015-2015MINAM), categoría 4 (Conservación de un ambiente acuático) debido a esto no se evidencio la presencia de peces, anfibios, reptiles ni mamíferos. Rico (2006) indica que el oxígeno en el agua es vital para la vida (peces, plantas, bacterias aerobias, macroinvertebrados, etc.), por ello la falta del mismo es dañina. Rodier (1988) menciona que la falta de oxígeno disuelto es un indicador de contaminación que puede estar en función de la presencia de productores y gérmenes aerobios, existencia de grasas, detergentes, entre otros.

Según Abarca (2007), la alta concentración de materia orgánica afecta negativamente los niveles de oxígeno disuelto en el agua, y si cae por debajo de $5.0 \mathrm{mg} / \mathrm{l}$, la vida acuática corre riesgo, al llegar a niveles menores a $2.0 \mathrm{mg} / \mathrm{l}$ los peces e invertebrados sufren mortandad porque el agua alcanzó condiciones de anoxia. La salinidad como segundo indicador resultó malo, indica que hay alteraciones por actividades antrópicas y los valores se desvían por encima del $50 \%$ de lo esperado en su estado natural (Rico, 2006). El pH, fue 6.8 aunque pueden existir variaciones y limitar la posibilidad de vida acuática.

La disponibilidad de fósforo fue mayor a $150 \mu \mathrm{g} / \mathrm{l}$ aumentó en $\mathrm{pH}$ básico, el fósforo es el factor limitante de la productividad primaria y además posee un efecto mayor sobre la eutrofización, puesto que cantidades mínimas de este elemento pueden favorecer al crecimiento de fitoplancton, afectando la estructura y funcionamiento del ecosistema acuático. Debido a la actividad humana, existe un aumento en la cantidad de fósforo en diversos cuerpos de agua, esto es producido por fertilizantes, detergentes, descargar industriales y humanas (Roldán, 2008).

El nitrógeno total fue mayor a $2000 \mu \mathrm{g} / \mathrm{l}$, representa un indicador de contaminación por desechos fecales, el nivel de amoniaco se incrementa por las prácticas agrícolas que utilizan fertilizantes de amonio y excretas de ganado que llegan a las fuentes de agua (Flores, 2007).

Los indicadores microbiológicos son representados por los coliformes termotolerantes, estos al sobrepasar valores de ECA indican contaminación fecal debido a que proceden del intestino humano y animales de sangre caliente. Su presencia evidencia el riesgo en el control de calidad del agua, mientras más coliformes se concentran en el agua, mayor es la gravedad de la descarga de heces (Tucto, 2014).

El estado ecológico general del humedal urbano Phuyuqocha - Cajonahuaylla es malo, por ello las aguas no alcanzan un buen estado ecológico y químico, para proteger la salud humana, el suministro, los ecosistemas naturales y la biodiversidad de acuerdo al sistema de indicadores ecológicos, basado en las características morfométricas, físicas, químicas, microbiológicas y en las comunidades bióticas del ecosistema acuático de la Directiva Marco del Agua de la Unión Europea (2006).Entonces se puede verificar que la causa principal de la disminución de los parámetros ecológicos de los humedales es por efecto de la intervención antrópica por falta de educación ambiental de la población y compromiso ambiental de sus autoridades.

Paredes (1998), identifica las principales actividades antrópicas vinculadas al proceso de eutrofización como son: construcción del muro-cerco del estadio municipal de Cajonahuaylla, construcción de un acceso vehicular a la sede de la policía ecológica, construcción de la vía asfaltada CuscoUrcos, paradero final de vehículos de transporte urbano, construcción de viviendas en sus alrededores, depósito de residuos sólidos y aguas servidas. Actualmente según las observaciones realizadas, el humedal, aún sigue recibiendo la descarga de residuos sólidos, se observa un crecimiento urbanístico acelerado sin planificación, se evidencia la presencia de animales domésticos, es lugar de eliminación de neumáticos y es un lugar de crianza de gansos domésticos. 
Rico (2006), indica la presencia de zonas urbanas afecta considerablemente los humedales, ocasionando una transformación de su estado ecológico y conduciendo a que desaparezca con el transcurso del tiempo. La urbanización altera los humedales por medio de cambios en la conectividad hidrológica, alteraciones de hábitats y las capas freáticas, asî́ como la saturación del suelo y contaminación, esto puede afectar también la riqueza y abundancia de especies (Faulkner, 2004). Teniendo en cuenta el desarrollo urbano de las ciudades y la fuerte presión sobre las zonas de reserva.

\section{CONCLUSIÓN}

El estado ecológico del humedal urbano Phuyuqocha Cajonahuaylla, considerando todos los indicadores evaluados, es calificado como malo, producto de la actividad antrópica que afecta directamente el cuerpo de agua.

\section{REFERENCIAS BIBLIOGRÁFICAS}

Abarca J.F. (2007). Técnicas para evaluación y monitoreo del estado de los humedales y otros ecosistemas acuáticos. Phoenix, Arizona.

Flores, S. (2007). Algas del Humedal Lucre-Huacarpay. (Seminario de Investigación). Universidad Nacional de San Antonio Abad del Cusco, Perú.

Faulkner, S. (2004). Urbanization impacts on the structure and function of forested wetlands. Urban Ecosystems 7, 89-106.
Paredes, O. (1998). Expansión urbana y efecto en el humedal Cajonahuaylla. Perú. Editorial Universitaria-UNSAAC.

Rico, E. (2006). Red de seguimiento de humedales interiores de la CAPV (2005-2006). Universidad Autónoma de Madrid. Red de seguimiento de humedales interiores de la CAPV.ingurumeda.net

Rodier, J. 1978. Análisis de las aguas. Ed. Omega, Barcelona. Roldán, G. (2008). Fundamentos de Limnológia neotropical. Segunda Ed..Universidad de Antioquia. Medellin -Colombia, Ruza, R. (2008). Indicadores biológicos utilizados en el control $\mathrm{y}$ vigilancia de la calidad de las aguas.

Samanez I. (2014). Métodos de colecta, identificación y análisis de comunidades biológicas: plancton, perifiton, bentos (macroinvertebrados) y necton (peces) en aguas continentales del Perú. Primera edición, diciembre 2014, Lima - Perú.

Secretaría de la Convención de Ramsar (2018). Manual de la Convención de Ramsar: Guía a la Convención sobre los Humedales (Ramsar, Irán, 1971), 4a. edición. Secretaría de la Convención de Ramsar, Gland (Suiza).

Tucto, R. (2014). Limnología y nivel trófico de la laguna de Urcos-Cusco. (Tesis para optar el título profesional de Biólogo). Universidad San Antonio Abad del Cusco, Perú.

Presentado: $27 / 04 / 2021$

Aceptado: $15 / 08 / 2021$

Publicado: 31/01/2022 